\title{
Title: Identification of novel human USP2 inhibitor: might involve in SARS- CoV-2 papain-like (PLpro) protease deubiquitination activity
}

Short running title: Human USP2 inhibitor against SARS-CoV-2 protease

Muhammad Usman Mirza ${ }^{1}$, Sarfraz Ahmad ${ }^{2}$, Iskandar Abdullah ${ }^{2}$, Matheus Froeyen ${ }^{1}$

${ }^{1}$ Department of Pharmaceutical and Pharmacological Sciences, Rega Institute for Medical Research, Medicinal Chemistry, University of Leuven, B-3000 Leuven, Belgium

${ }^{2}$ Department of Chemistry, Faculty of Science, University of Malaya, Kuala Lumpur 50603, Malaysia

\section{Corresponding author:}

Prof. Matheus Froeyen

Rega Institute for Medical Research,

Medicinal Chemistry, University of Leuven,

Belgium

Email: mathy.froeyen@kuleuven.be 


\title{
Highlights
}

- Inhibition of Jurkat and MOLT-4 cell-growth in low micromolar range $(\approx 10 \mu \mathrm{M})$ was achieved by a new human ubiquitin carboxyl-terminal hydrolase 2 (USP2) inhibitor (Z93).

- SARS-CoV-2 papain-like protease (PLpro) and USP2 share strikingly similar structural scaffold and conserved catalytic triad

- This is the first account in on-going COVID-19 outbreak where we purposed the identification of SARS-CoV-2 PLpro inhibitor with a proof of human USP2 inhibition.

\begin{abstract}
The ubiquitin-specific protease 2 (USP) belongs to the family of deubiquitinases and plays a critical role in tumors cells' survival and therefore signifies an important therapeutic target. Previous studies have indicated promising efficacies of potent human USP2 inhibitors including, thiopurine analogues against SARS-CoV papain-like proteases (PLpro). The PLpro have significant functional implications in the innate immune response during SARS-CoV-2 infection and considered an important antiviral target. Both proteases share strikingly similar USP fold with right-handed thumb-palm-fingers structural scaffold and conserved catalytic triad Cys-His-Asp/Asn. In this urgency situation of COVID-19 outbreak, there is a lack of in-vitro facilities readily available to test SARS-CoV-2 inhibitors in whole-cell assays. Therefore, we adopted an alternate route to identify potential USP2 inhibitor through integrated structure-based virtual screening efforts. After a subsequent virtual screening protocol, the best compounds were selected and tested. The compound $\mathrm{Z} 93$ showed significant $\mathrm{IC}_{50}$ value against Jurkat $(9.67 \mu \mathrm{M})$ and MOTL-4 cells $(11.8$ $\mu \mathrm{M})$. The binding mode of Z93 was extensively analyzed through molecular docking, followed by MD simulations, and molecular interactions were compared with SARS-CoV-2. The relative binding poses of Z93 fitted well in the binding site of both proteases and showed consensus $\pi$ - $\pi$ stacking and H-bond interactions with histidine and aspartate/asparagine residues of the catalytic triad. These results led us to speculate that compound Z93 might be the first potential chemical lead against SARS-CoV-2 PLpro, which warrants in-vitro evaluations.
\end{abstract}

Keywords: Deubiquitination, leukemia, Ubiquitin-specific protease 2 (USP2), SARS-CoV-2 papain-like protease (PLpro), COVID-19

\section{Introduction}

Leukemia is a hematological malignancy originate in the bone marrow and results in abnormally increased production of improperly functioning white blood cells. It causes prognosis based acute or chronic illness commonly associated with lymphocyte or myeloid cells. With the growing availability of treatment options, the morbidity of acute lymphoblastic leukemia (ALL) is responsible for mortality in 20\% pediatric and $50 \%$ adult cases. T-cell associated ALL (T-ALL) is aggressive from of ALL with a prevalence of $15 \%$ pediatric and $25 \%$ adult patients ${ }^{1}$.

Deubiquitinating peptidases (DUBs) are a broad class of proteases that dissociate ubiquitin from proteins and disrupt proteasomal and lysosomal degradation of its substrate proteins. Many members of DUB have found to play their role in the progression of different cancers ${ }^{2}$. Such as, USP2 is specific deubiquitinase of a proto-oncoprotein cyclin D1 and stabilizes it via restriction of ubiquitin-dependent degradation ${ }^{3}$. This affirms that the type of cancers which depend on cyclin D1 for their growth, e.g., T-ALL ${ }^{4}$, can be suppressed by using USP2 inhibitors.

The active site of human USP2 shares a similar architecture with SARS-CoV-2 PLpro, which has deubiquitinating activity and directs important implications on viral replication and pathogenesis ${ }^{5}$. The 
structural analysis revealed a similar active site of SARS-CoV-2 PLpro, including the signature catalytic triad (Cys-His-Asp/Asn) located at the interface of the thumb and Palm sub-domains ${ }^{6}$. Various studies have reported the efficacy of USP2 potent inhibitors including thiopurine analogs on SARS 7,8 and MERS ${ }^{9}$ which were found to interact with the signature catalytic triad ( $\mathrm{IC}_{50}$ values ranges from 5 to $21.6 \mu \mathrm{M}$ ). With the recent COVID-19 outbreak which resulted in more than 126,000 deaths worldwide ${ }^{10}$, there is a dearth of in-vitro facilities available to test SARS-CoV-2 inhibitors in whole-cell assays. Therefore, an alternate route might be the selective inhibition of USP2 by small molecules which may plausibly lead to the identification of SARS-CoV-2 PLpro inhibitors due to structurally conserved similar active site fold. Based on these assumptions, the present study focuses on the identification of potential USP2 inhibitor through in-depth structure-based virtual screening workflow. The computational work was carried out in sequential way followed by molecular dynamics (MD) simulation and interaction energetics using AMBER simulation package. The identified compounds were purchased and tested on Jurkat and MOLT-4 cells against T-ALL. The binding mode analysis was extensively performed to examine the molecular interactions together with SARS-CoV-2 PLpro. To the best of our knowledge, the utilized method is the first study reported in on-going COVID-19 outbreak where we propose SARS-CoV-2 PLpro inhibitor with a proof of human USP2 inhibition based on highly similar structural attributes.

\section{Material and methods}

\section{Data collection and docking structure preparation}

For the structure-based study, the co-crystalized structure of human USP2 complex with 6-thioguanine (5XU8) was utilized for docking. The structure was prepared accordingly, as described previously ${ }^{11,12}$. Briefly, all heteroatoms were removed, hydrogens and charges were added into USP2. The structure was minimized for the 1000 steepest descent steps at root means square gradient of 0.02 having an update interval of 10 and using the AMBER ff14SB force field. A chemical library of $\sim 4.2$ million molecules was retrieved from ZINC. The library was reduced after applying various drug-like parameter, which mainly includes the Lipinski's Rule of five (with 2 violations), oral bioavailability rule including: rotatable bonds $\leq 10$ and TPSA $\leq 140 \AA^{2}$. The resulted dataset was utilized for structure-based virtual screening workflow.

\section{Virtual screening workflow}

Before subsequent docking runs, the docking grid was defined by selecting the specific area around the catalytic triad residues (Cys276, His557, and Asn574). The filtered database was finally uploaded into the Mcule drug discovery platform and AutoDock Vina was utilized to screen the docking library ${ }^{13}$. Diversity filter was also applied in workflow, which reduced the docking library by removing the closest analogs and maximized the coverage of chemical space to identify active scaffolds. Autodock Vina was selected among the top-ranked scoring functions in terms of docking power and screening test according to CASF (Comparative Assessment of Scoring Functions) benchmark $2016^{14}$. The compounds were ranked accordingly on docking score which is based on the Vina empirical scoring function that approximates the ligand binding affinity in $\mathrm{kcal} / \mathrm{mol}$. The top hits were analysed for ADMET criteria and top hits were analysed in Chimera in terms of binding poses and interactions with the catalytic triad. The overall stability of the best complexes was analysed over a period of 20ns using AMBER 18 simulation package ${ }^{15}$. We used the same MD simulation protocol as described previously ${ }^{12,16}$. Briefly, the Antechamber was utilized to generate the general AMBER force field (GAFF) parameters for the studied ligands. After a stepwise minimization, heating and equilibration in explicit solvent environment (TIP3), a production run of 20ns was performed at standard pressure $(\mathrm{p}=1.0 \mathrm{~atm})$ and temperature $(\mathrm{T}=300 \mathrm{~K})$. The binding free energies $\left(\Delta \mathrm{G}_{\mathrm{tol}}\right)$ of USP2 complexed with screened compounds were calculated using the MM-GBSA method of AMBER 18. The compounds with significant interaction profile were purchased based on availability and 
price. The tested compounds were later redocked using AD Vina on recently resolved crystal structure of SARS-CoV-2 PLpro (6W9C) and compared the molecular interactions. For molecular docking, docking grid was setup around the catalytic triad (Cys112, His272 and Asp286). The same MD simulation protocol was utilized for SARS-CoV-2 PLpro complexes.

\section{In Vitro cytotoxicity}

\section{Materials}

Jurkat and MOLT-4 cells were used to inspect the cytotoxicity of the selected compound against T-ALL. The test compound and cisplatin were purchased from MolPort (Life Chemicals Inc.). Cell culture media, fetal calf serum (FCS), antibiotic/antimycotic, MTT (3-[4-C-dimethylthiazol-2-yl]-2,5-diphenyltetrazolium bromide) and accompanying materials were purchased from Thermo Fisher Scientific, Inc, (Waltham, MA, USA).

\section{MTT Assay}

The cells were cultured in RPMI-1640 medium accompanied with 10\% heat-inactivated FCS, $100 \mu \mathrm{g} / \mathrm{ml}$ streptomycin and $100 \mathrm{U} / \mathrm{ml}$ penicillin and incubated in a humidified atmosphere at $37^{\circ} \mathrm{C}$ and $5 \% \mathrm{CO}_{2}$. The cell suspension was adjusted to a final concentration of $3 \times 10^{5} \mathrm{cells} / \mathrm{ml}$ and $100 \mu \mathrm{l}$ cell suspension dispensed per well. The plate was incubated at $37{ }^{\circ} \mathrm{C}$ for $12 \mathrm{~h}$ in $5 \% \mathrm{CO}_{2}$ incubator. The stock solutions $(10 \mathrm{mM})$ of the test compound and cisplatin were prepared in DMSO and the test concentrations were prepared by diluting the stocks with the growth media. The test dilutions $(100 \mu \mathrm{l})$ were added to the wells giving final concentrations of $1,5,10,20$ and $40 \mu \mathrm{M}$. MTT reagent $(20 \mu 1,5 \mathrm{mg} / \mathrm{ml}$ in PBS) was added to each microwell after $48 \mathrm{~h}$, followed by incubation for $5 \mathrm{~h}$. The contents were solubilized with $100 \mu \mathrm{l} 10 \%$ sodium dodecylsulfate in $10 \mathrm{mM} \mathrm{HCl}$. The absorbance was measured after $12 \mathrm{~h}$ with the help of BioRad PR 4100 microplate reader at a wavelength of $570 \mathrm{~nm}$. Percentage viability was calculated by using following relation, and dose-response curves were plotted. The results represent mean \pm SD of six readings; three determinations of two independent experiments ${ }^{11,17}$.

$$
\text { Percentage viability }=\left[\frac{A s-A b}{A c-A b}\right] \times 100
$$

Where, As, Ab and Ac are absorbance of sample, blank and control, respectively.

\section{Results}

\section{Virtual screening workflow validation using set of known USP2 binders}

To validate the docking protocol, a total of 12 reported USP2 inhibitors were subjected to an established virtual screening pipeline. The $\mathrm{IC}_{50}$ values of inhibitors against USP2 were converted to $\mathrm{pIC}_{50}$ values $(-\log$ of $\mathrm{IC}_{50}$ ) and plotted against the $\mathrm{AD}$ Vina docking scores and MMGBSA total binding free energy scores calculated by Amber-mmgbsa method. The corresponding values are listed in Table $\mathbf{S 1}$ and correlation plot between $\mathrm{pIC}_{50}$ values and docking scores is displayed in Figure 1. The AD Vina docking scores showed weak correlation with the experimental $\mathrm{pIC}_{50}$ values $\left(R^{2}=0.21\right)$, whereas MMGBSA scores showed better correlation $\left(R^{2}=0.53\right)$. Here, we speculated that the weak correlation between AD Vina docking scores and experimental $\mathrm{pIC}_{50}$ values might be indorsed by side chains conformational flexibility of binding site residues, which were adjusted during MD simulations in explicit solvent environment. Hence, the MMGBSA total binding free energy showed better correlation with experimental values, as it incorporates all electrostatic and van der Waals (vdW) interactions. Overall, combining AD Vina followed by 
MMGBSA calculations suggested a reliable approach to select the compounds against USP2 (together with expected false positives and negatives).
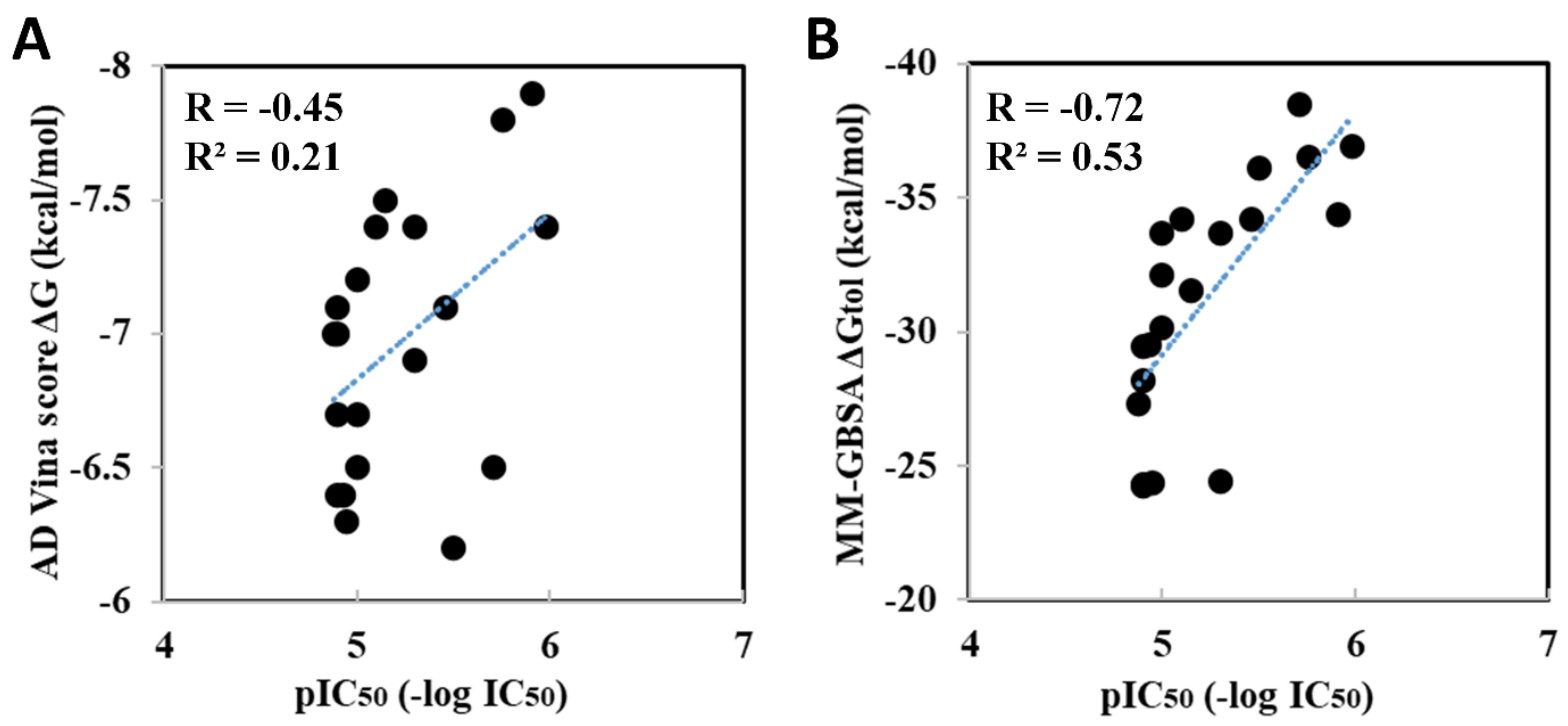

Figure 1: Correlation between the AutoDock Vina docking score and IC50 for 20 known human-USP2 binders. (A) Plot of AD Vina docking score against pIC50. (B) Plot of MMGBSA value (total binding free energy calculated after 5ns MD simulations) against pIC50.

\section{Virtual screening}

To identify potential inhibitors against UPS2, structure-based virtual screening was employed. A substantial number of compounds were eliminated through several ADMET filters, which include drug-like and toxicity assessment. A total of 52 compounds were selected based on the AD Vina score from a known USP2 binder (CHEMBL3392809, AD Vina score: $-7.3 \mathrm{kcal} / \mathrm{mol}$ ) as cut-off value. Among these hits, each compound was investigated for molecular interactions with signature catalytic triad (Cys276, His557, and Asn574), which further reduced the list to 14 compounds. The final selection of compounds was made after extensive MD simulation analysis of these 14 complexes which included, 1) constant interaction with any of the catalytic triad residues, 2) lowest MMGBSA total binding free energy values and 3) stable conformation of ligand over a period of $20 \mathrm{~ns}$ (Figure S1). A total of 3 compounds were finally selected and only one compound (ZINC9325709 denoted as Z93) was purchased for biological evaluations based on the specific scaffold, its availability and price (Figure 2 and Table S2). The detailed ADMET properties are tabulated in (Table S3). 


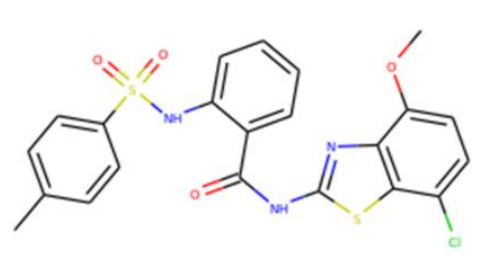

ZINC9325709 (Z93)<smiles>COc1cc(NS(=O)(=O)c2cccc(C)c2)cc2sc(NC(=O)c3ccccc3)nc12</smiles>

ZINC33008019<smiles>CC1C(=O)c2cc(NC(=O)CCc3cccc4ccccc34)ccc2C(C)C1=O</smiles>

ZINC68861734<smiles>Cc1ccc(S(=O)(=O)Nc2ccccc2C(=O)Nc2nc(-c3ccccc3)c(Br)s2)cc1</smiles>

CHEMBL3392809

Figure 2: Chemical structure of selected compounds from in silico screen. CHEMBL3392809, potent USP2 inhibitor is included as reference.

\section{Biological evaluation}

Jurkat and MOLT-4 cell lines were used to examine cytotoxicity of selected USP2 inhibitor (Z93) against T-ALL while cisplatin was used as a control. Both selected cell lines display versatile characteristics to study cytotoxicity of drugs against T-ALL. The dose-response curve is presented in Figure 3. The $I C_{50}$ value of Z93 against Jurkat and MOTL-4 cells were 9.67 and $11.8 \mu \mathrm{M}$, respectively, after $48 \mathrm{~h}$ of exposure. Under same conditions cisplatin depicted 16.88 and $20.01 \mu \mathrm{M} I C_{50}$ values against Jurkat and MOLT-4, respectively.

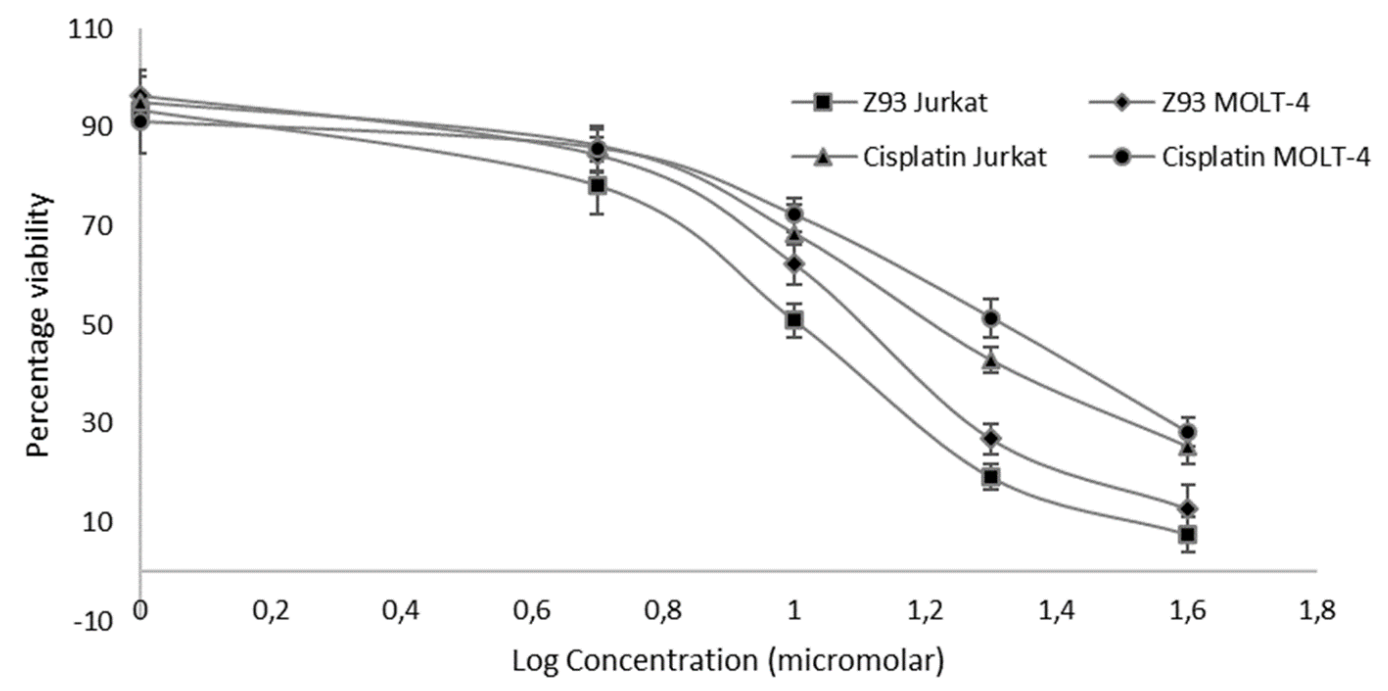

Figure 3: Dose-response curve for Z93 and cisplatin against Jurkat and MOLT-4 cell lines. 


\section{Binding mode of Z93 with USP2 and SARS-CoV-2 PLpro}

Recent studies have determined the crystal structure of MERS, SARS-CoV and CoV-2 PLpro, which corroborated the similar palm-thumb-fingers structural scaffold and conserved catalytic triad Cys-HisAsn/Asp like in human USP2, albeit the low sequence homology ${ }^{6,18,19}$. After superimposition, the corresponding domain architecture of both PLpro displayed strikingly similar representation of conserved USP fold and orientation of catalytic triad residues (Figure 4A- 4D). These findings led us to speculate that identified Z93, a potential USP2 inhibitor might be able to inhibit the SARS-CoV-2 PLpro because both proteases share a similar catalytic triad. Overall, the integrated virtual screening identified 14 hits which were reduced to 3 after analysing the binding free energies (Figure 4E).

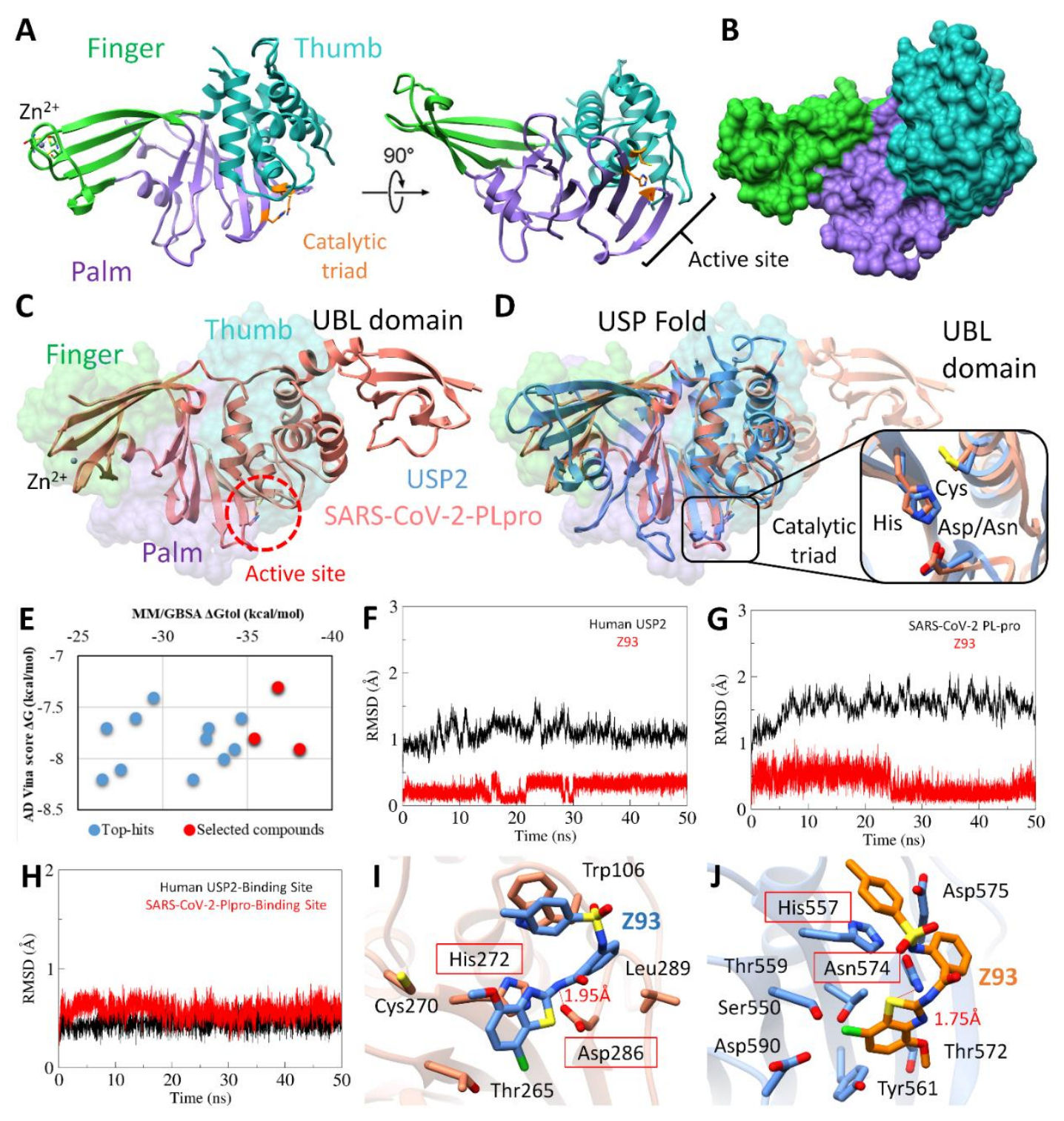

Figure 4: Molecular modelling of human USP2 and SARS-CoV-2 papain-like protease (PLpro). (A) Overall structure of the USP12 comprises of finger (green), palm (purple) and thumb (sea green) domains. The catalytic center (Cys box) is shown in orange between the palm and thumb domains. (B) Surface representation of the structure of the human USP2. (C) The SARS-CoV-2 PLpro monomer (PDB: 6W9C) consists of four domains: beginning from $\mathrm{N}$ - to the C-terminus, the extended UBL, the thumb, palm and 
fingers domain. The active site is circled red. (D) An overlay of SARS-CoV-2 PLpro (salmon) with USP2 (cornflower blue) displaying the conserved USP fold. The catalytic triad residues are magnified. (E) The energy plot of top hits between corresponding AD Vina docking score and MMGBSA total binding free energy calculated after a production run of $20 \mathrm{~ns}$ MD simulations). The selected compounds are highlighted red. Root-mean-square-deviation of USP2 (F) and SARS-CoV-2 PLpro (G) backbone are estimated over a period of 20 ns with bound Z93 ligand (red). (H) RMSD plot of binding site residues of corresponding proteases. The MD simulated binding modes of Z93 (sticks representation) inside the active site of USP2 (I) and SARS-CoV-2 PLpro (J).

To gain further insight into the binding mode, we performed molecular docking followed by MD simulations against both PLpro and most favourable binding conformation of active compounds, Z93 was obtained from the largest cluster after 20ns production run through clustering analysis. The RMSD plot indicated the stable backbone conformation of binding site residues with docked Z93 (Figure 4F). Overall, both complexes fluctuated in the start and remained stable for last $20 \mathrm{~ns}$ indicated that Z93 adopted more favourable conformation (Figure 4G and $\mathbf{4 H}$ ). Despite the similar docked sites on both PLpro, the binding poses were found different. More insights revealed the consensus binding interactions of Z93 with the catalytic triad in both PLpro. In UPS2, the major interaction was established due to stable stacking interaction between terminal benzene of Z93 and His557 of catalytic triad. Moreover, the benzothiazole moiety of Z93, apart from establishing a H-bond with the side chain nitrogen atom of Asn574 also formed extensive hydrophobic interactions with Thr572, Thr559, Ser550 and Tyr561 (Figure 4I). In relation to SARS-CoV-2 PLpro, the Z93 also found interacted with the catalytic triad where the benzothiazole moiety was positioned to make direct stacking interaction with His272, and terminal benzene was positioned to form stacking interaction with Trp106 (Figure 4J). Additionally, the nitrogen atom next to benzothiazole established H-bond with the sidechain oxygen atom of conserved Asp286 of catalytic triad. The predicted binding site was close to that found for SARS-CoV PLpro ${ }^{7}$.

Overall, the stable binding mode of Z93 in both proteases was mainly governed due to stacking interactions and complexes were remained converged with an RMSD value of less than $0.5 \AA$. Moreover, the estimated total binding energies after $20 \mathrm{~ns}$ MD simulation for Z93 binding to USP2 and SARS-CoV-2 PL pro were -38.07 and $-33.05 \mathrm{kcal} / \mathrm{mol}$ respectively. Z93 participated well through vdW interactions as evident from strong stacking interactions with relatively less electrostatic contributions (Table S4).

\section{Discussion}

Ubiquitin carboxyl-terminal hydrolase 2 (USP2) is a highly conserved protein present in most eukaryotes. It is found to be involved in intracellular proteasomal degradation, cell-cycle regulation and stress response. The overexpression of USP2 has been found involved in progression and metastasis several cancers, e.g., triple negative breast cancer, colorectal cancer and non-Hodgkin's lymphoma ${ }^{20}$. Most of the proteasome inhibitors usually pose extensive off-target effects and an alternative approach of USP2 inhibition can selectively target proteasomal degradation of its multiple oncogenic substrates with least effect on noncancerous cells ${ }^{21,22}$.

It antagonizes ubiquitin-dependent degradation of cyclin D1 resulting in its stabilization. The cells involving cyclin D1 independent cell-cycle progress, for example, normal human fibroblasts or cancer cells that don't show cyclin D1 expression are not affected by the inactivation of USP2. These evidences suggest that inhibition of USP2 in cancer cells which express cyclin D1 for their growth could induce growth suppression ${ }^{3}$. ML364, an already reported inhibitor of USP2, has close structural similarity with Z93, with both sharing 2-((4-methylphenyl)sulfonamido)benzamide residue. ML364 induces cyclin D1 degradation 
resulting in cell-cycle arrest, indicating key role in progression of non-Hodgkin's lymphoma and colorectal cancer ${ }^{22}$. Cyclin D1 is necessarily involved in cell-cycle progression from G1 to $\mathrm{S}$ phase and its overexpression is evident in many human cancers ${ }^{23}$. It is found to be upregulated in most of the ALL patient while its expression is not affected in AML patients as compared to the normal controls ${ }^{4}$. Reduced survival rates have been observed in ALL patients with higher expression of cyclin D1, suggesting its involvement in the progression of aggressive forms of $\mathrm{ALL}^{23}$.

6-Mercaptopurine (6MP) and 6-thioguanine (6TG) has been reported effective against the treatment of various cancer including leukemia. These both compounds are hypoxanthine-guanine phosphoribosyltransferase inhibitors and possess purine residue ${ }^{24}$. Another potential effect of these compounds have been reported against MERS-CoV and SARS-CoV PLpro ${ }^{9}$. The benzothiazole moiety of Z93 resembles 6MP and 6TG purine, having [4.3.0] bicyclic system. In another study, indole based 3chymotrypsin-like protease (3CLpro) inhibitor of SARS-CoV have been reported with low micromolar range ${ }^{25}$. These analogies strongly potentiate the hypothesis that Z93 can inhibit SARS-CoV-2 by antagonizing its PLpro.

The modelling studies indicated the consensus molecular interactions governed by Z93 with both proteases. There was no major structural deviation observed over a period of $20 \mathrm{~ns}$ in both docked complexes. With both proteases, Z93 interacted with the His and Asp/Asn of catalytic triad in a similar way, albeit with different binding mode and revealed favourable total binding free energies. The Z93 bound conformation was found more stable with SARS-CoV-2 PLpro due to an additional staking interaction with Trp106, therefore showed more favourable vdW energy contributions, as compared to Z93/USP2. Conclusively, based on the in-vitro and repurposed modelling analysis, it is entirely possible to develop selective, noncovalent inhibitors against SARS-CoV-2 PLpro by using structure-based approach against USPs involved in disease processes.

\section{Acknowledgements}

The authors are grateful to Rega Institute for Medical Research and Department of Chemistry, Malaya University (Grant Number: IIRG003A-2019) for providing necessary facilities to accomplish this study.

\section{Conflict of Interest}

The authors declare no conflict of interest

\section{Authors contributions}

M.U.M and M.F conceived and designed the experiments. M.U.M. performed the in-silico studies. M.U.M. S.A. and I.A performed experiments. M.F supervised the study. M.U.M. wrote the main manuscript text. S.A, I.A and M.F critically reviewed the manuscript. All the authors approved the final manuscript. 


\section{References}

1. Vadillo E, Dorantes-Acosta E, Pelayo R, Schnoor M. T cell acute lymphoblastic leukemia (TALL): New insights into the cellular origins and infiltration mechanisms common and unique among hematologic malignancies. Blood Reviews. 2018;32(1):36-51.

2. Young M-J, Hsu K-C, Lin TE, Chang W-C, Hung J-J. The role of ubiquitin-specific peptidases in cancer progression. Journal of Biomedical Science. 2019;26(1):42.

3. Shan J, Zhao W, Gu W. Suppression of cancer cell growth by promoting cyclin D1 degradation. Molecular Cell. 2009;36(3):469-476.

4. Aref S, Mabed M, El-Sherbiny M, Selim T, Metwaly A. Cyclin D1 expression in acute leukemia. Hematology. 2006;11(1):31-34.

5. Barretto N, Jukneliene D, Ratia K, Chen Z, Mesecar AD, Baker SC. The papain-like protease of severe acute respiratory syndrome coronavirus has deubiquitinating activity. Journal of virology. 2005;79(24):15189-15198.

6. Chou CY, Lai HY, Chen HY, Cheng SC, Cheng KW, Chou YW. Structural basis for catalysis and ubiquitin recognition by the severe acute respiratory syndrome coronavirus papain-like protease. Acta Crystallographica Section D: Biological Crystallography. 2014;70(2):572-581.

7. Chou C-Y, Chien C-H, Han Y-S, et al. Thiopurine analogues inhibit papain-like protease of severe acute respiratory syndrome coronavirus. Biochemical pharmacology. 2008;75(8):1601-1609.

8. Chen X, Chou C-Y, Chang G-G. Thiopurine analogue inhibitors of severe acute respiratory syndrome-coronavirus papain-like protease, a deubiquitinating and deISGylating enzyme. Antiviral Chemistry and Chemotherapy. 2009;19(4):151-156.

9. Cheng K-W, Cheng S-C, Chen W-Y, et al. Thiopurine analogs and mycophenolic acid synergistically inhibit the papain-like protease of Middle East respiratory syndrome coronavirus. Antiviral research. 2015;115:9-16.

10. World Health Organization. https://www.who.int/docs/default-source/coronaviruse/situationreports/20200414-sitrep-85-covid-19.pdf?sfvrsn=7b8629bb_4. Accessed 15.04.2020.

11. Ikram N, Mirza MU, Vanmeert M, et al. Inhibition of oncogenic kinases: An in vitro validated computational approach identified potential multi-target anticancer compounds. Biomolecules. 2019;9(4).

12. Mirza MU, Vanmeert M, Froeyen M, Ali A, Rafique S, Idrees M. In silico structural elucidation of RNA-dependent RNA polymerase towards the identification of potential Crimean-Congo Hemorrhagic Fever Virus inhibitors. Scientific reports. 2019;9(1):1-18.

13. Kiss R, Sandor M, Szalai FA. http://Mcule. com: a public web service for drug discovery. Journal of cheminformatics. 2012;4(S1):P17.

14. Su M, Yang Q, Du Y, et al. Comparative assessment of scoring functions: The CASF-2016 update. Journal of chemical information and modeling. 2018;59(2):895-913.

15. Case DA, Ben-Shalom IY, Brozell SR, et al. AMBER 2018; 2018. University of California, San Francisco.

16. Mirza MU, Rafique S, Ali $\mathrm{A}$, et al. Towards peptide vaccines against Zika virus: Immunoinformatics combined with molecular dynamics simulations to predict antigenic epitopes of Zika viral proteins. Scientific reports. 2016;6:37313.

17. Papa V, Tazzari PL, Chiarini F, et al. Proapoptotic activity and chemosensitizing effect of the novel Akt inhibitor perifosine in acute myelogenous leukemia cells. Leukemia. 2008;22(1):147-160.

18. Báez-Santos YM, John SES, Mesecar AD. The SARS-coronavirus papain-like protease: structure, function and inhibition by designed antiviral compounds. Antiviral research. 2015;115:21-38.

19. Lei J, Mesters JR, Drosten C, Anemüller S, Ma Q, Hilgenfeld R. Crystal structure of the papainlike protease of MERS coronavirus reveals unusual, potentially druggable active-site features. Antiviral research. 2014;109:72-82.

20. Qu Q, Mao Y, Xiao G, et al. USP2 promotes cell migration and invasion in triple negative breast cancer cell lines. Tumor Biology. 2015;36(7):5415-5423. 
21. Halford B. From Discovery To Drug. Chemical \& Engineering News. 2012;90(35):34-35.

22. Davis MI, Pragani R, Fox JT, et al. Small molecule inhibition of the ubiquitin-specific protease USP2 accelerates cyclin D1 degradation and leads to cell cycle arrest in colorectal cancer and mantle cell lymphoma models. Journal of Biological Chemistry. 2016;291(47):24628-24640.

23. Fernandes JC, Alves APNR, Coelho-Silva JL, et al. Increased levels of cyclin D1 negatively impacts on acute lymphoblastic leukemia overall survival. Applied Cancer Research. 2018;38(1):7.

24. Pieters R, Huismans DR, Loonen AH, et al. Hypoxanthine-guanine phosphoribosyl-transferase in childhood leukemia: Relation with immunophenotype, in vitro drug resistance and clinical prognosis. International journal of cancer. 1992;51(2):213-217.

25. Turlington M, Chun A, Tomar S, et al. Discovery of N-(benzo [1, 2, 3] triazol-1-yl)-N-(benzyl) acetamido) phenyl) carboxamides as severe acute respiratory syndrome coronavirus (SARS-CoV) 3CLpro inhibitors: identification of ML300 and noncovalent nanomolar inhibitors with an inducedfit binding. Bioorganic \& Medicinal Chemistry Letters. 2013;23(22):6172-6177. 